Ji Li* and Siqi Wu

\title{
The crystal structure of dichlorido-(tris(2-benzimidazolylmethyl)amine- $\left.\mathrm{K}^{4} N, N^{\prime}, N^{\prime \prime}, N^{\prime \prime \prime}\right)$ chromium(III) chloride - methanol (1/3), $\mathrm{CrC}_{27} \mathrm{H}_{33} \mathrm{Cl}_{3} \mathrm{~N}_{7} \mathrm{O}_{3}$
}

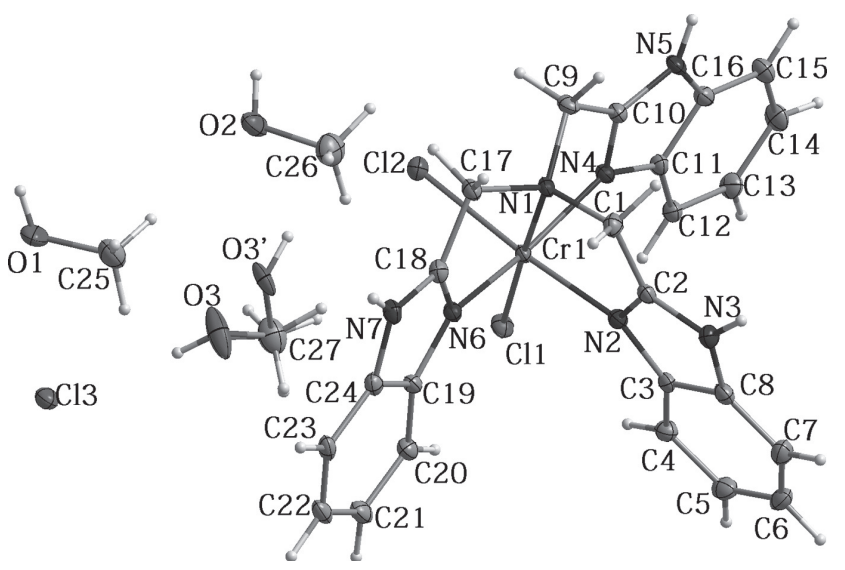

https://doi.org/10.1515/ncrs-2019-0607

Received August 21, 2019; accepted November 11, 2019; available online December 5, 2019

\section{Abstract}

$\mathrm{CrC}_{27} \mathrm{H}_{33} \mathrm{Cl}_{3} \mathrm{~N}_{7} \mathrm{O}_{3}$, triclinic, $P \overline{1}$ (no. 2), $a=9.7862$ (13) $\AA$, $b=12.4690(17) \AA, \quad c=12.8413(17), \quad \alpha=84.163(2)^{\circ}$, $\beta=73.602(3)^{\circ}, \quad \gamma=85.524(2)^{\circ}, \quad V=1493.4(3) \AA^{3}, \quad Z=2$, $R_{\mathrm{gt}}(F)=0.0472, w R_{\text {ref }}\left(F^{2}\right)=0.1524, T=100(2) \mathrm{K}$.

\section{CCDC no.: 1947955}

The asymmetric unit of the title crystal structure is shown in the figure. Table 1 contains crystallographic data and Table 2 contains the list of the atoms including atomic coordinates and displacement parameters.

\section{Source of materials}

Tris(2-benzimidazolylmethyl)amine (NTB) was prepared according to an earlier reported method [7]. NTB (0.408 g, $1.0 \mathrm{mmol})$ was dissolved in hot methanol $(50.0 \mathrm{~mL})$ and then a solution of $\mathrm{CrCl}_{3}(0.158 \mathrm{~g}, 1.0 \mathrm{mmol})$ in $20.0 \mathrm{~mL}$ methanol was added drop by drop. The mixed solution was stirred for $30 \mathrm{~min}$ at $50{ }^{\circ} \mathrm{C}$. Finally, the reaction mixture was filtered and the filtrate solution was evaporated at room temperature

\footnotetext{
*Corresponding author: Ji Li, School of Biological and
} Environmental Engineering, Guiyang University, Guiyang 550005, P.R. China, e-mail: Liji_guiyang@126.com. https://orcid.org/00000003-1878-6585

Siqi Wu: School of Biological and Environmental Engineering, Guiyang University, Guiyang 550005, P.R. China
Table 1: Data collection and handling.

\begin{tabular}{ll}
\hline Crystal: & Block, green \\
Size: & $0.15 \times 0.12 \times 0.10 \mathrm{~mm}$ \\
Wavelength: & Mo $K \alpha$ radiation $(0.71073 \AA$ A) \\
$\mu:$ & $0.69 \mathrm{~mm}^{-1}$ \\
Diffractometer, scan mode: & Bruker APEX-II, $\varphi$ and $\omega$-scans \\
$\theta_{\text {max }}$, completeness: & $28.3^{\circ},>99 \%$ \\
$N(h k l)_{\text {measured }}, N(h k l)_{\text {unique }}, R_{\text {int }}:$ & $13443,7221,0.036$ \\
Criterion for $I_{\text {obs }}, N(h k l)_{\text {gt }}:$ & $I_{\text {obs }}>2 \sigma\left(I_{\text {obs }}\right), 5873$ \\
$N(\text { param })_{\text {refined }}:$ & 391 \\
Programs: & Bruker programs [1], SHELX [2-4], \\
& DIAMOND [5], OLEX2 [6] \\
\hline
\end{tabular}

Table 2: Fractional atomic coordinates and isotropic or equivalent isotropic displacement parameters $\left(\AA^{2}\right)$.

\begin{tabular}{|c|c|c|c|c|}
\hline Atom & $x$ & $y$ & $z$ & $\boldsymbol{U}_{\text {iso }} * / \boldsymbol{U}_{\text {eq }}$ \\
\hline r1 & $0.87932(4)$ & $0.24696(3)$ & $0.35537(3)$ & $0.01037(12)$ \\
\hline 1 & $0.5572(2)$ & $0.10643(17)$ & $1.08021(17)$ & $0.0294(5)$ \\
\hline 11 & $0.597(4)$ & $0.0454(16)$ & $1.087(3)$ & $0.044^{*}$ \\
\hline 02 & $0.9564(2)$ & $0.19453(16)$ & $0.86432(15)$ & $0.0232(4)$ \\
\hline $\mathrm{H} 2$ & $1.030(2)$ & $0.157(2)$ & $0.866(3)$ & $0.035^{\star}$ \\
\hline $03^{a}$ & $0.4809(3)$ & $0.1332(2)$ & $0.7181(2)$ & $0.0396(8)$ \\
\hline$H 3 A^{a}$ & 0.4251 & 0.1419 & 0.7799 & $0.059^{*}$ \\
\hline $3^{\prime b}$ & $0.5987(13)$ & $0.1141(7)$ & $0.6653(9)$ & $0.0396(8)$ \\
\hline $13^{\prime b}$ & 0.6877 & 0.1025 & 0.6473 & 0.059 * \\
\hline 11 & $0.82188(6)$ & $0.39065(4)$ & $0.45741(4)$ & $0.01619(14)$ \\
\hline 12 & $0.91378(6)$ & $0.13139(5)$ & $0.50164(4)$ & $0.01610(14)$ \\
\hline 13 & $0.23008(6)$ & $0.09169(5)$ & $0.92587(5)$ & $0.01804(14)$ \\
\hline V1 & $0.9336(2)$ & $0.11276(15)$ & $0.25366(15)$ & $0.0117(4)$ \\
\hline N2 & $0.8550(2)$ & $0.32773(15)$ & 76(16) & $127(4)$ \\
\hline V3 & $0.8637(2)$ & $0.31986(16)$ & $0.03942(16)$ & $0.0151(4)$ \\
\hline H3 & 895(3) & $0.294(2)$ & $-0.0247(13)$ & $0.018^{*}$ \\
\hline 14 & $1.0931(2)$ & $0.26305(15)$ & $0.29001(16)$ & $0.0123(4)$ \\
\hline$\sqrt{5}$ & $1.3009(2)$ & $0.19395(16)$ & $0.19269(16)$ & $0.0148(4)$ \\
\hline $\mathrm{H} 5 \mathrm{~A}$ & $1.367(2)$ & $0.1476(19)$ & $0.161(2)$ & $0.018^{*}$ \\
\hline N6 & $0.6845(2)$ & $0.18585(15)$ & $0.38087(15)$ & $0.0129(4)$ \\
\hline N7 & $0.5594(2)$ & $0.04882(16)$ & $0.36851(16)$ & $0.0145(4)$ \\
\hline $\mathrm{H} 7 \mathrm{~A}$ & $0.534(3)$ & $-0.0141(13)$ & $0.358(2)$ & $0.017^{*}$ \\
\hline $\mathrm{C} 1$ & $0.9177(3)$ & $0.14815(18)$ & $0.14170(19)$ & $0.0152(5)$ \\
\hline $\mathrm{H} 1 \mathrm{~A}$ & 1.0085 & 0.1323 & 0.0862 & $0.018^{*}$ \\
\hline H1B & 0.8428 & 0.1072 & 0.1278 & $0.018^{*}$ \\
\hline $\mathrm{C} 2$ & $0.8787(2)$ & $0.26562(19)$ & $0.13211(18)$ & $0.0127(4)$ \\
\hline $\mathrm{C} 3$ & $0.8258(2)$ & $0.43219(18)$ & $0.17069(18)$ & $0.0126(4)$ \\
\hline $\mathrm{C} 4$ & $0.7961(3)$ & $0.53062(19)$ & $0.2178(2)$ & $0.0159(5)$ \\
\hline H4 & 0.7916 & 0.5353 & 0.2921 & 0.019 * \\
\hline $\mathrm{C} 5$ & $0.7736(3)$ & $0.6211(2)$ & $0.1513(2)$ & $0.0195(5)$ \\
\hline
\end{tabular}


Table 2 (continued)

\begin{tabular}{|c|c|c|c|c|}
\hline Atom & $x$ & $y$ & $z$ & $\boldsymbol{U}_{\text {iso }}{ }^{*} / \boldsymbol{U}_{\text {eq }}$ \\
\hline H5 & 0.7541 & 0.6893 & 0.1807 & $0.023^{*}$ \\
\hline $\mathrm{C} 6$ & $0.7787(3)$ & $0.6146(2)$ & $0.0420(2)$ & $0.0207(5)$ \\
\hline H6 & 0.7624 & 0.6786 & -0.0007 & $0.025^{\star}$ \\
\hline $\mathrm{C7}$ & $0.8067(3)$ & $0.5180(2)$ & $-0.0053(2)$ & $0.0199(5)$ \\
\hline $\mathrm{H} 7$ & 0.8094 & 0.5135 & -0.0793 & $0.024^{\star}$ \\
\hline C8 & $0.8309(3)$ & $0.4276(2)$ & $0.0608(2)$ & $0.0156(5)$ \\
\hline C9 & $1.0847(2)$ & $0.07642(19)$ & $0.2481(2)$ & $0.0148(5)$ \\
\hline H9A & 1.1276 & 0.0364 & 0.1829 & $0.018^{*}$ \\
\hline H9B & 1.0890 & 0.0287 & 0.3137 & $0.018^{*}$ \\
\hline C10 & $1.1626(2)$ & $0.17676(18)$ & $0.24190(18)$ & $0.0132(4)$ \\
\hline C11 & $1.1930(2)$ & $0.34181(19)$ & $0.27076(19)$ & $0.0133(4)$ \\
\hline $\mathrm{C} 12$ & $1.1792(3)$ & $0.44687(19)$ & $0.30174(19)$ & $0.0147(4)$ \\
\hline $\mathrm{H} 12$ & 1.0905 & 0.4770 & 0.3428 & $0.018^{*}$ \\
\hline C13 & $1.3013(3)$ & $0.5055(2)$ & $0.2697(2)$ & $0.0185(5)$ \\
\hline H13 & 1.2961 & 0.5770 & 0.2904 & $0.022^{\star}$ \\
\hline C14 & $1.4323(3)$ & $0.4623(2)$ & $0.2076(2)$ & $0.0209(5)$ \\
\hline H14 & 1.5126 & 0.5059 & 0.1859 & $0.025^{\star}$ \\
\hline C15 & $1.4469(3)$ & $0.3571(2)$ & $0.1772(2)$ & $0.0197(5)$ \\
\hline H15 & 1.5357 & 0.3270 & 0.1364 & $0.024^{\star}$ \\
\hline C16 & $1.3246(3)$ & $0.29844(19)$ & $0.20976(19)$ & $0.0148(5)$ \\
\hline C17 & $0.8306(2)$ & $0.02783(18)$ & $0.30757(19)$ & $0.0142(4)$ \\
\hline $\mathrm{H} 17 \mathrm{~A}$ & 0.8242 & -0.0223 & 0.2543 & $0.017^{\star}$ \\
\hline H17B & 0.8616 & -0.0142 & 0.3674 & $0.017^{*}$ \\
\hline C18 & $0.6898(3)$ & $0.08606(19)$ & $0.35124(18)$ & $0.0139(4)$ \\
\hline C19 & $0.5399(2)$ & $0.21641(19)$ & $0.42022(18)$ & $0.0127(4)$ \\
\hline $\mathrm{C} 20$ & $0.4731(3)$ & $0.3126(2)$ & $0.46055(19)$ & $0.0159(5)$ \\
\hline $\mathrm{H} 2 \mathrm{O}$ & 0.5263 & 0.3721 & 0.4639 & $0.019^{\star}$ \\
\hline C21 & $0.3258(3)$ & $0.3171(2)$ & $0.4954(2)$ & $0.0189(5)$ \\
\hline $\mathrm{H} 21$ & 0.2767 & 0.3810 & 0.5243 & $0.023^{\star}$ \\
\hline $\mathrm{C} 22$ & $0.2464(3)$ & $0.2297(2)$ & $0.48932(19)$ & $0.0189(5)$ \\
\hline H22 & 0.1453 & 0.2364 & 0.5138 & $0.023^{\star}$ \\
\hline C23 & $0.3115(3)$ & $0.1343(2)$ & $0.44872(19)$ & $0.0162(5)$ \\
\hline H23 & 0.2579 & 0.0753 & 0.4449 & $0.019^{\star}$ \\
\hline $\mathrm{C} 24$ & $0.4607(3)$ & $0.12970(19)$ & $0.41353(18)$ & $0.0140(4)$ \\
\hline $\mathrm{C} 25$ & $0.5900(3)$ & $0.1465(3)$ & $0.9689(2)$ & $0.0290(6)$ \\
\hline $\mathrm{H} 25 \mathrm{~A}$ & 0.5302 & 0.2120 & 0.9611 & $0.043^{*}$ \\
\hline $\mathrm{H} 25 \mathrm{~B}$ & 0.5717 & 0.0917 & 0.9256 & $0.043^{*}$ \\
\hline $\mathrm{H} 25 \mathrm{C}$ & 0.6907 & 0.1636 & 0.9431 & $0.043^{*}$ \\
\hline C26 & $0.9777(3)$ & $0.2404(2)$ & $0.7549(2)$ & $0.0297(6)$ \\
\hline $\mathrm{H} 26 \mathrm{~A}$ & 0.9376 & 0.3149 & 0.7552 & $0.045^{\star}$ \\
\hline $\mathrm{H} 26 \mathrm{~B}$ & 0.9301 & 0.1980 & 0.7165 & $0.045^{\star}$ \\
\hline $\mathrm{H} 26 \mathrm{C}$ & 1.0801 & 0.2398 & 0.7179 & $0.045^{\star}$ \\
\hline $\mathrm{C} 27$ & $0.5644(3)$ & $0.2223(2)$ & $0.6994(2)$ & $0.0305(6)$ \\
\hline $\mathrm{H} 27 \mathrm{~A}^{\mathrm{a}}$ & 0.5039 & 0.2877 & 0.6930 & $0.046^{*}$ \\
\hline $\mathrm{H} 27 \mathrm{~B}^{\mathrm{a}}$ & 0.6411 & 0.2166 & 0.6317 & $0.046^{*}$ \\
\hline $\mathrm{H} 27 \mathrm{C}^{\mathrm{a}}$ & 0.6038 & 0.2246 & 0.7612 & $0.046^{\star}$ \\
\hline$H 27 D^{b}$ & 0.6496 & 0.2644 & 0.6723 & $0.046^{*}$ \\
\hline $\mathrm{H} 27 \mathrm{E}^{\mathrm{b}}$ & 0.4885 & 0.2563 & 0.6692 & $0.046^{*}$ \\
\hline $\mathrm{H} 27 \mathrm{~F}^{\mathrm{b}}$ & 0.5335 & 0.2196 & 0.7791 & $0.046^{*}$ \\
\hline
\end{tabular}

Occupancies: ${ }^{\mathrm{a}} 0.800(4),{ }^{\mathrm{b}} 0.200(4)$. for ten days. Some green block crystals were obtained at the bottom of the vessel $(0.50 \mathrm{~g}$, yield: $75 \%)$.

\section{Experimental details}

Hydrogen atoms bonded to carbon atoms were placed in their geometrically idealized positions and constrained to ride on their parent atoms with $\mathrm{C}-\mathrm{H}=0.95 \AA$ (aromatic), $0.99 \AA$ Å (methylene), $0.98 \AA$ (methyl) $U_{\text {iso }}(\mathrm{H})=1.2 U_{\text {eq }}$ (aromatic and methylene) and $1.5 U_{\text {eq }}$ (methyl). Hydrogen atoms bonded to nitrogen, $\mathrm{O} 1$ and $\mathrm{O} 2$ atoms were initially found from the difference maps and refined with the restraints of $\mathrm{N}-\mathrm{H}=0.88(1) \AA, \mathrm{O}-\mathrm{H}=0.84(1) \AA$. The positions of hydrogen atoms bonded to disordered 03 and $\mathrm{O3}^{\prime}$ were constrained to be at the 'best' positions, forming hydrogen bonds to neighboring chloride anions [3]. Commands DFIX and SADI were used in the refinement to restrain $\mathrm{C}-\mathrm{O}, \mathrm{N}-\mathrm{H}$ and $\mathrm{O}-\mathrm{H}$ distances, and EADP was used to restrain the thermal factor of $\mathrm{O3}^{\prime}$ being similar to $\mathrm{O} 3$. For the $\mathrm{N}$ and $\mathrm{O}$ atoms, $U_{\text {iso }}(\mathrm{H})=1.2 U_{\text {eq }}(\mathrm{N})$ and $1.5 U_{\text {eq }}(\mathrm{O})$.

\section{Comment}

The multi-benzimidazole ligand tris(2-benzimidazolylmethyl) amine (NTB) has been often used in the design and synthesis of metal-containing compounds due to their potential application in the fields of biological and bioinorganic chemistry [8-10]. NTB has been often seen to be a tetradentate ligand coordinating a late transition metal. Compared to these metal complexes, early transition metal complexes of NTB are rarely reported.

The asymmetric unit of the title structure is composed of each one $\left[\mathrm{Cr}(\mathrm{NTB}) \mathrm{Cl}_{2}\right]^{+}$, one $\mathrm{Cl}^{-}$anion and three methanol solvent molecules. NTB acts as a tetradentate ligand, coordinating through the tertiary $\mathrm{N}$ atom and three benzimidazole (bzim) $\mathrm{N}$ atoms to metal ion. Two chlorido ligands are in a cis configuration. The $\mathrm{N}_{4} \mathrm{Cl}_{2}$ donor set constructs an octahedral polyhedron around the central $\mathrm{Cr}(\mathrm{III})$. The tertiary $\mathrm{N} 1$ atom presents a bond length about 0.10 Å longer than those for the three bzim $\mathrm{N}$ atoms. To retain the octahedral geometry, ligand NTB adopts a planar geometry with a dihedral angle of 11.9(1) ${ }^{\circ}$ between the N4/N5 and N6/N7-containing bzim groups. However, the angles of these moieties with the third one are 79.9(1) and $88.3(1)^{\circ}$, respectively, which are very similar to its analogous structure with different solvent [11]. By considering some NTB-containing analogs as a whole, it can be found NTB can adopt many spatial arrangements to meet the coordination preference of a metal ion or solvent molecules [12-14]. On the other hand, the $\mathrm{Cr}(\mathrm{III})$ ion lies in the plane formed by N1, N4, N6 and CL1 atoms (maximal deviation: $0.025 \AA$ for Cr1). As a result of these features, the deviations from an ideal octahedral geometry around $\mathrm{Cr}$ (III) ion are the following: trans 
angles range from $158.09(8)$ to $178.29(5)^{\circ}$ and cis angles from 78.92(7) to $101.19(6)^{\circ}$.

A detailed geometrically analysis using the program PLATON [15] indicates that these adjacent $\left[\mathrm{Cr}(\mathrm{NTB}) \mathrm{Cl}_{2}\right]^{+}$ions are firstly linked into two-dimensional layers parallel to the (011) plane by three $\pi \cdots \pi$ interactions. These layers are further linked by three $\mathrm{N}-\mathrm{H} \cdots \mathrm{O}$ and three $\mathrm{O}-\mathrm{H} \cdots \mathrm{Cl}$ hydrogen bonds into the final three-dimensional network.

Acknowledgements: This work was financially supported by Discipline and Master's Site Construction Project of Guiyang University by Guiyang City Financial Support Guiyang University (SH-2019).

\section{References}

1. Bruker. SMART and SAINT. Bruker AXS Inc., Madison, WI, USA (2003).

2. Sheldrick, G. M.: A short history of SHELX. Acta Crystallogr. A64 (2008) 112-122.

3. Sheldrick, G. M.: SHELXTL Integrated space-group and crystal-structure determination. Acta Crystallogr. A71 (2015) 3-8.

4. Sheldrick, G. M.: Crystal refinement with SHELX. Acta Crystallogr. C71 (2015) 3-8.

5. Brandenburg, K.: DIAMOND. Crystal Impact GbR, Bonn Germany (2006).

6. Dolomanov, O. V.; Bourhis, L. J.; Gildea, R. J.; Howard, J. A. K.; Puschmann, H.: OLEX2: a complete structure solution, refinement and analysis program. J. Appl. Cryst. 42 (2009) 339-341.
7. Szczepura, L. F.; Witham, L. M.; Takeuchi, K. J.: Tris(2-pyridyl) tripod ligands. Coord. Chem. Rev. 174 (1998) 5-32.

8. Desbouis, D.; Troitsky, I. P.; Belousoff, M. J.; Spiccia, L.; Graham, B.: Copper(II), zinc(II) and nickel(II) complexes as nuclease mimetics. Coord. Chem. Rev. 256 (2012) 897-937.

9. Barone, G.; Terenzi, A.; Lauria, A.; Almerico, A. M.; Leal, J. M.; Busto, N.; García, B.: DNA-binding of nickel(II), copper(II) and zinc(II) complexes: structure-affinity relationships. Coord. Chem. Rev. 257 (2013) 2848-2862.

10. Tsou, C. C.; Yang, W. L.; Liaw, W. F.: Nitrite activation to nitric oxide via one-fold protonation of iron(II)-0,0-nitrito complex: relevance to the nitrite reductase activity of deoxyhemoglobin and deoxyhemerythrin. J. Am. Chem. Soc. 135 (2013) 18758-18761.

11. Ceniceros-Gómez, A. E.; Barba-Behrens, N.; Quiroz-Castro, M. E.; Bernès, S.; Nöth, H.; Castillo-Blum, S. E.: Synthesis, X-ray and spectroscopic characterisation of chromium(III) coordination compounds with benzimidazolic ligands. Polyhedron 19 (2000) 1821-1827.

12. Chen, J.; Zhang, H. D.: Crystal structure of dinitrato-k $O$-bis (tris((1H-benzo[d]imidazol-2-yl)methyl)amine- $\left.{ }^{4} N, N^{\prime}, N^{\prime \prime}, N^{\prime \prime \prime}\right)$ ( $\mu_{2}$-cyclohexane-1,4-dicarboxylato- $\left.{ }^{4} O, O^{\prime}: O^{\prime \prime}, O^{\prime \prime \prime}\right)$ dimanganese(II)-methanol-water (1/6/2), $\mathrm{C}_{62} \mathrm{H}_{80} \mathrm{Mn}_{2} \mathrm{~N}_{16} \mathrm{O}_{18}$. Z. Kristallogr. NCS 232 (2017) 775-777.

13. Chen, J.; Zhang, L. C.; Chen, J. W.; Zhang, H. D.: Crystal structure of [tris(2-benzimidazolylmethyl)amine- $\left.{ }^{4} N, N^{\prime}, N^{\prime \prime}, N^{\prime \prime \prime}\right]$ [(pyridine-2,6-dicarboxylato- $\mathrm{K}^{2} \mathrm{O}, \mathrm{N}$ )] cadmium(II)-methanol (1:3) $\mathrm{C}_{34} \mathrm{H}_{36} \mathrm{CdN}_{8} \mathrm{O}_{7}$. Z. Kristallogr. NCS 233 (2018) 1047-1049.

14. Jang, J. J.; Li, L.; Yang, T.; Kuang, D. B.; Wang, W.; Su, C. Y.: Self-assembly of 2D Borromean networks through hydrogen-bonding recognition. Chem. Comm. (2009) 2387-2389.

15. Spek, A. L.: Structure validation in chemical crystallography. Acta Crystallogr. D65 (2009) 148-155. 